EXTENDED REPORT

\title{
In vivo evaluation of ocular inflammatory responses in experimental diabetes
}

\author{
H Tamura, J Kiryu, K Miyamoto, K Nishijima, H Katsuta, S Miyahara, F Hirose, Y Honda, \\ N Yoshimura
}

See end of article for authors' affiliations

....................

Correspondence to:

Junichi Kiryu, MD,

Department of

Ophthalmology and Visual

Sciences, Kyoto University

Graduate School of

Medicine, Sakyo, Kyoto

606-8507, Japan;

kiryu@kuhp.kyoto-u.ac.jp

Accepted for publication

1 January 2005
Aims: Diabetic patients may have abnormal inflammatory reactions to foreign or endogenous stimuli. This study was designed to evaluate inflammatory reactions in the diabetic eye through retinal leucocyte dynamics in the inflamed eyes of diabetic rats.

Methods: Three weeks after diabetes induction in Long-Evans rats, endotoxin induced uveitis was produced by footpad injection of lipopolysaccharide (LPS). After LPS injection, leucocyte behaviour was evaluated in vivo by acridine orange digital fluorography.

Results: The number of rolling leucocytes increased in a biphasic manner at 12 hours and 48 hours. The number of leucocytes accumulating in the retina reached a peak at 72 hours. The maximal numbers of rolling and accumulating leucocytes in the diabetic retina decreased by $56.3 \%(p<0.01)$ and $46.7 \%$ $(p<0.0001)$, respectively, compared with the non-diabetic retina. The levels of mRNA expression of adhesion molecules in the retina, which were upregulated after LPS injection, were also lower in diabetic rats than in non-diabetic rats.

Conclusion: This study is the first to show that endotoxin induced inflammation is disturbed in the diabetic eye, based on evidence that the leucocyte-endothelial cell interactions stimulated by LPS were suppressed in the diabetic retina. These findings support the theory that ocular inflammatory reactions are impaired in diabetic patients. eucocytes have a crucial role in inflammation by interacting with endothelial cells, migrating to the sites of

-inflammation and releasing cytokines. In an inflamed area, endothelial cells are activated and express adhesion molecules that cause leucocyte-endothelial cell interactions through a multistep process. ${ }^{12}$ Initially, leucocytes interact with P-selectin, which is expressed on endothelial cells, and begin rolling along vessel walls. The leucocytes then interact with intercellular adhesion molecule 1 (ICAM-1), adhere to endothelial cells, and migrate out of the vessels.

The inflammatory reaction in diabetic patients, one of compromised hosts, is thought to be abnormal. Under diabetic conditions, reduced cytokine production ${ }^{3}$ and depressed chemotaxis during inflammation have been described. ${ }^{4-6}$ The available literatures suggest that leucocyteendothelial cell interactions during inflammation, being one of the inflammatory processes and an important initial step, are disturbed in various organs in diabetes. ${ }^{4-9}$ However, little is known about leucocyte-endothelial cell interactions in the inflamed diabetic eyes, even though the diabetic retinopathy is one of the major complications of diabetes.

Endotoxin induced uveitis (EIU) is an ocular inflammation model induced in an animal by a subcutaneous injection of lipopolysaccharide (LPS), ${ }^{10}$ without direct exposure to the eye. ${ }^{11}{ }^{12}$ It is characterised by leucocyte infiltration, bloodocular disruption, and barrier disruption. In this model, inflammatory reactions have been reported to occur in both the anterior and posterior segments of the eye, including infiltration of leucocytes into the aqueous humour $(\mathrm{AH})$ and vitreous cavity. ${ }^{11-15}$

In the EIU, the expression of tumour necrosis factor alpha $(\mathrm{TNF}-\alpha)$ is elevated. TNF- $\alpha$ has been proposed as a major mediator of endotoxin induced reactions, since changes caused by infusions of TNF- $\alpha$ in laboratory animals mimic those observed in animals infected with Gram negative bacteria. ${ }^{16}{ }^{17} \mathrm{TNF}-\alpha$ is involved early in the course of EIU. LPS is the most powerful TNF inducer of all sources tested to date. $^{18}$ Moreover, several investigators demonstrated that both P-selectin and ICAM-1 are regulated by TNF- $\alpha .{ }^{19}{ }^{19}$ During the EIU, the expression of inducible nitric oxide synthase (iNOS) is also upregulated, and supposed to have a key role in the pathogenesis of EIU. ${ }^{21}$ And nitric oxide (NO) derived from iNOS also regulates the expression of P-selectin and ICAM-1, ${ }^{22-24}$ and contributes to the vasodilation.

We have developed an in vivo method of quantitatively evaluating leucocyte-endothelial cell interactions in the rat retina during EIU, called acridine orange (AO) digital fluorography. ${ }^{14}{ }^{15} 25$ In this study, we investigated in rats the influence of streptozotocin induced diabetes on LPS stimulated leucocyte-endothelial cell interactions by quantitatively evaluating changes in leucocyte-endothelial cell interactions and changes in the expression of endothelial adhesion molecules and cytokines in the diabetic retina.

\section{METHODS}

\section{Animal model}

Animals $(n=108)$ were handled in accordance with the ARVO statement for the use of animals in ophthalmic and vision research. Diabetes mellitus (DM) was induced in 6 week old male pigmented Long-Evans rats by intraperitoneal injection of streptozotocin (STZ, $75 \mathrm{mg} / \mathrm{kg}$; Sigma Chemical Co, St Louis, MO, USA). The plasma glucose level in each rat was confirmed to be $>250 \mathrm{mg} / \mathrm{dl} 48$ hours after injection. Plasma glucose level was measured with Dexter Z II (Bayer Healthcare LLC, Terrytown, NY, USA). Rats injected with an equal volume of saline alone served as non-diabetic

Abbreviations: $\mathrm{AH}$, aqueous humour; $\mathrm{AO}$, acridine orange; $\mathrm{DM}$, diabetes mellitus; EIU, endotoxin induced uveitis; ICAM-1, intercellular adhesion molecule 1; iNOS, inducible nitric oxide synthase; LPS, lipopolysaccharide; NO, nitric oxide; PCR, polymerase chain reaction; STZ, streptozotocin; TNF- $\alpha$, tumour necrosis factor alpha 
Table 1 Physiological variables

\begin{tabular}{llllllll}
\hline \multicolumn{1}{l}{ Hours after LPS injection } \\
\cline { 2 - 8 } & Control & $\mathbf{6}$ hours & $\mathbf{1 2}$ hours & $\mathbf{2 4}$ hours & $\mathbf{4 8}$ hours & $\mathbf{7 2}$ hours & $\mathbf{9 6}$ hours \\
\hline Non-DM groups & $8.6(0.8)$ & $7.8(1.0)$ & $7.3(0.9)$ & $7.7(1.9)$ & $13.3(1.4)^{*}$ & $10.9(1.1)$ & $9.4(1.1)$ \\
WBC $\left(\times 10^{6} / \mathrm{l}\right)$ & $137(4)$ & $132(6)$ & $135(4)$ & $133(6)$ & $135(6)$ & $133(6)$ & $133(6)$ \\
Blood glucose $(\mathrm{mg} / \mathrm{ml})$ & $100(5)$ & $101(4)$ & $106(5)$ & $103(7)$ & $102(4)$ & $101(6)$ & $100(6)$ \\
MABP (mm Hg) & $8.1(0.3)$ & $6.7(1.3)$ & $7.4(0.8)$ & $7.7(0.5)$ & $11.1(0.5)^{*}$ & $9.6(0.9)$ & $8.7(1.2)$ \\
DM groups & $325(24) \dagger$ & $351(16) \dagger$ & $331(16) \dagger$ & $314(59) \dagger$ & $350(34) \dagger$ & $351(16) \dagger$ & $343(6) \dagger$ \\
WBC $\left(\times 10^{6} / \mathrm{l}\right)$ & $98(3)$ & $96(4)$ & $97(7)$ & $98(7)$ & $100(6)$ & $98(6)$ & $99(5)$ \\
Blood glucose $(\mathrm{mg} / \mathrm{ml})$ & MABP $(\mathrm{mm} \mathrm{Hg})$ & &
\end{tabular}

Values are means (SEM). ( $n=6$ at each time point in both groups)

$W B C$, peripheral leucocyte count; $M A B P$, mean arterial blood pressure.

Blood glucose level in diabetic rats was significantly higher than that in non-diabetic rats at each time point. There were no significant differences between nondiabetic and diabetic rats in other physiological parameters. ${ }^{*} p<0.05$ compared with control values in each group. $t p<0.05$ compared with values of non-

diabetic rats at each time point.

(non-DM) controls. Three weeks after diabetes induction, EIU was induced by hind footpad injection of LPS (Salmonella typhimurium $4 \mathrm{mg} / \mathrm{kg}$ of body weight; Sigma Chemical Co, St Louis, MO, USA).

\section{Evaluation of blood pressures and leucocyte count in peripheral blood}

The rats were deeply anaesthetised and the mean arterial blood pressure was monitored at each time point in the tail of each rat by means of a sensing cuff attached to a blood pressure analyser (IITC, Woodland Hills, CA, USA). The peripheral blood sample was analysed by a haematology analyser to count leucocytes (Erma, Tokyo, Japan).

\section{Acridine orange digital fluorography and image analysis}

At 6, 12, 24, 48, 72, and 96 hours after LPS injection, inflammatory leucocyte behavior was evaluated in vivo by AO digital fluorography using a scanning laser ophthalmoscope (SLO) (Rodenstock Instruments, Munich, Germany). ${ }^{14} 25$

Vessel diameters were measured at 1 disc diameter from the centre of the optic disc in monochromatic images recorded before AO injection. Each vessel diameter was calculated in pixels as the distance between the half height points determined separately on both sides of the density profile of the vessel image. Averages of the arterial and venous diameters were used as the arterial and venous diameters for each rat.

Rolling leucocytes were defined as leucocytes that moved more slowly than free flowing leucocytes. To count rolling leucocytes, a locus 1 disc diameter away from the centre of the optic disc was picked up in each vessel. The rolling leucocytes passing through this locus during 1 minute were counted, and this count was defined as the number of rolling leucocytes within a given vessel. We then calculated the average number of rolling leucocytes in all major veins. This was used as the flux of rolling leucocytes within a given rat.

To define the number of leucocytes that accumulated in the retinal microcirculation, an observation area around the optic disc was determined by drawing a polygon surrounded by the adjacent major retinal vessels. ${ }^{25}$ This area was measured in pixels on a computer monitor, and the density of leucocytes was calculated by dividing the number of trapped leucocytes, which were recognised as fluorescent dots, by the area of observation. The density of leucocytes was calculated in eight peripapillary observation areas in the fundus. The average density for individual areas was used as the number of leucocytes that had accumulated in the retinal microcirculation for each rat.

\section{Gene expression of P-selectin, ICAM-1, TNF- $\alpha$, and iNOS}

For semiquantification of P-selectin, ICAM- 1, TNF- $\alpha$, and iNOS gene expression after 9 hours of LPS injection, six eyes per group were enucleated in the following four groups: control rats (non-DM, without EIU), non-DM rats with EIU, DM rats with EIU, and DM rats without EIU. Polymerase chain reaction (PCR) was performed as previously described. $^{26}$ The primers were TGCTTGGCTACTGGACACTG (sense) and GGTGTCGACAGGACATTGTG (antisense) for Pselectin; AGCCTCAGGCCTAAGAGGAC (sense) and AGGGG TCCCAGAGAGGTCTA (antisense) for ICAM-I; TGATCCGA GATGGAACTG (sense) and TGTGGGTGAGGAGCACATAG (antisense) for TNF- $\alpha$; AGTGAGGAGCAGGTTGAGGA (sense) and CTTCAGTCGGGTGGTTCATT (antisense) for iNOS; GGCATCCTGACCCTGAAGTA (sense) and GCCATCTC TTGCTCGAAGTC (antisense) for $\beta$ actin was used as the internal standard.

\section{Analysis of leucocyte infiltration into $\mathrm{AH}$}

Aqueous humour $(\mathrm{AH})$ was collected by anterior chamber puncture using a 30 gauge needle. For leucocyte counts, the sample was suspended in an equal volume of $0.4 \%$ trypan blue stain solution, and leucocytes were counted under a light microscope.

\section{Statistical analysis}

All values are expressed as mean (SEM). The data were analysed by repeated measure ANOVA, with post hoc comparisons tested with the Fisher protected least significant difference procedure. Differences were considered statistically significant when the probability values were $<0.05$.

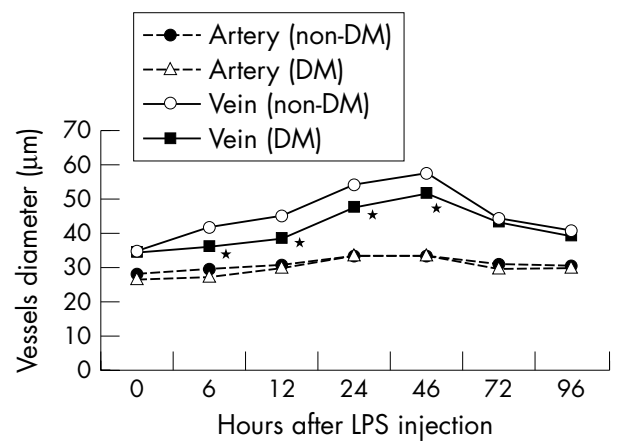

Figure 1 Time course of the diameters of major retinal arteries and veins after LPS injection. Values are mean (SEM) ( $n=6$ at each time point in both groups). " $p<0.05$ compared with non-DM rats. 

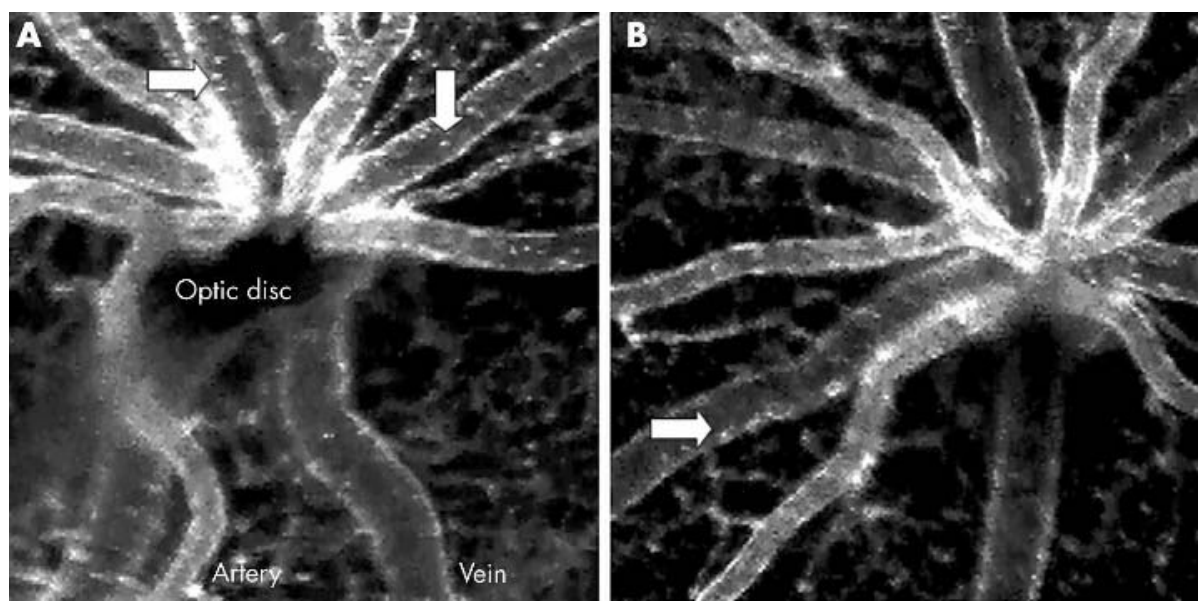

Figure 2 Leucocytes were stained selectively among circulation blood cells. Nuclei of vascular endothelial cells were also stained. Arrows indicate rolling leucocytes in the major retinal veins. No rolling leucocytes were seen along the major arteries. The major retinal veins of non-DM rats (A) were more dilated and more tortuous than those of DM rats (B) at 48 hours.

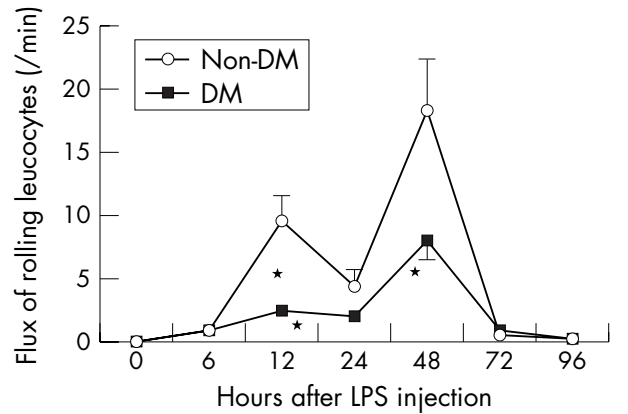

Figure 3 Time course of the flux of rolling leucocytes along major retinal veins after LPS injection. Values are mean (SEM) $(n=6$ at each time point in both groups). ${ }^{*} p<0.05$ compared with non-DM rats.

\section{RESULTS}

\section{Physiological data}

Table 1 shows changes in physiological variables at various time points after LPS injection. There were no significant differences between the DM groups and non-DM groups in peripheral leucocyte count and mean arterial blood pressure except for plasma glucose level.

\section{Major retinal vessel diameters}

Figure 1 shows changes in diameters of major retinal vessels in DM rats and non-DM rats. Both arteries and veins exhibited substantial vasodilation after EIU induction, and reached a peak at 48 hours. However, there was no significant difference between DM rats and non-DM rats in arterial vasodilation, while vasodilation of the veins was significantly reduced in DM rats, compared with non-DM rats $(\mathrm{p}=0.046$, fig 2$)$.

\section{Leucocyte rolling}

Immediately after AO was infused intravenously, leucocytes were stained selectively among the circulating blood cells. In EIU rats, numerous leucocytes were observed to be rolling slowly along major retinal veins among many free flowing leucocytes. No rolling leucocytes were detected in control rats, but leucocyte rolling was evident in major retinal veins in rats with EIU. The flux of rolling leucocytes in EIU rats increased gradually after LPS injection and reached its peaks at 12 hours and 48 hours. Figure 3 shows that leucocyte rolling was significantly inhibited in DM rats, compared with non-DM rats $(p=0.023)$. The flux of rolling leucocytes in DM rats was reduced by $73.0 \%(p=0.009)$ and $56.3 \%(p=0.006)$ at 12 hours and 48 hours, respectively, after LPS injection.
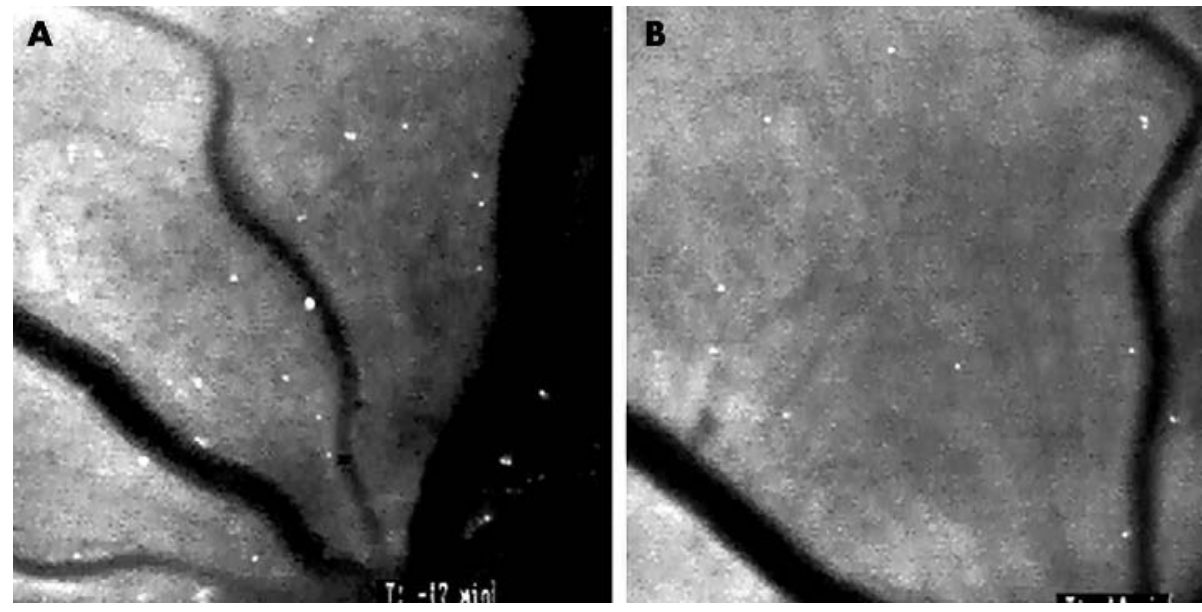

Figure 4 Leucocytes accumulating in the retina were observed as fluorescent dots at 30 minutes after $A O$ injection. (A) Non-DM rats; (B) DM rats. Significant reduction of leucocyte accumulation was seen in the DM rats (B) at 72 hours after LPS injection. 


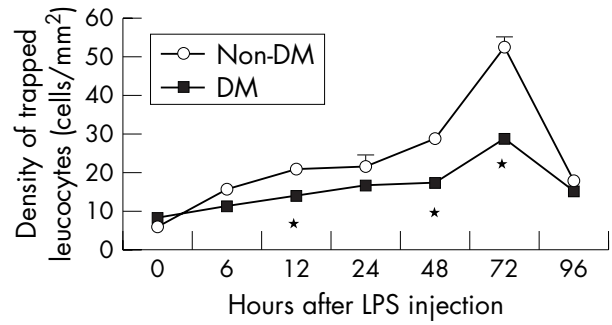

Figure 5 Time course of the number of leucocytes accumulating in the retinal microcirculation after LPS injection. Values are mean (SEM) $(n=6$ at each time point in both groups). ${ }^{*} p<0.05$ compared with non-DM rats.

\section{Leucocyte accumulation}

At 30 minutes after AO injection, we identified leucocytes that had accumulated in the retinal microcirculation as distinct fluorescent dots with the highest contrast. With the induction of EIU, the number of leucocytes that accumulated in the retina increased, and peaked at 72 hours (fig 4). However, the number of leucocytes that had accumulated in the retinal microcirculation decreased by $46.7 \%$ in DM rats compared with non-DM rats $(\mathrm{p}<0.0001$, fig 5$)$.

\section{Gene expression of P-selectin, ICAM-1, TNF- $\alpha$, and iNOS}

The levels of gene expression were expressed as ratios to the mean values of control rats (fig 6). At 9 hours after LPS injection, mRNA expression of adhesion molecules such as Pselectin and ICAM-1 was upregulated in the retina. The mRNA expression TNF- $\alpha$, which is a mediator of the above mentioned adhesion molecules, was also upregulated. The presence of DM significantly suppressed mRNA expression for P-selectin, ICAM-1, TNF- $\alpha$, and iNOS by $50.0 \%$ $(\mathrm{p}=0.038), \quad 52.6 \% \quad(\mathrm{p}=0.025), \quad 73.5 \% \quad(\mathrm{p}=0.006), \quad$ and $35.0 \%(\mathrm{p}=0.006)$, respectively.

\section{Leucocyte infiltration into $\mathrm{AH}$}

Figure 7 shows differences in the number of AH leucocytes between DM and non-DM groups. Leucocyte counts in AH gradually increased after EIU induction and reached a peak at 24 hours. In DM rats, however, leucocyte counts in $\mathrm{AH}$ were significantly lower than in non-DM rats $(p<0.0001)$. At 24 hours after LPS injection, the DM appeared to depress leucocyte infiltration in AH by $34.0 \%(p<0.0001)$.

\section{DISCUSSION}

This study has shown that leucocyte-endothelial cell interactions are impaired in the inflamed eye of diabetic rats. In addition, mRNA expression of P-selectin, ICAM- 1 , TNF- $\alpha$, and iNOS in the EIU retina was significantly suppressed, and leucocyte infiltration into the $\mathrm{AH}$ was also suppressed in the diabetic EIU model. To our knowledge, this is the first report of defective leucocyte-endothelial cell interactions in inflamed diabetic eyes.

Accumulating evidence indicates that recruitment of circulating leucocytes into inflamed tissue is mediated through a multistep cascade of events involving sequential rolling, firm adhesion, and transmigration. Leucocyte rolling is the initial step and prerequisite for the subsequent steps that result in leucocyte accumulation in the retinal microcirculation. ${ }^{27}$ Previous reports have shown that leucocyteendothelial cell interactions are impaired and that the subsequent leucocyte rolling and accumulation are reduced in the lung and internal spermatic fascia of diabetic rats. ${ }^{478}$ We have already reported that diabetic retina shows suppressed leucocyte-endothelial cell interactions after ischaemia reperfusion injury. ${ }^{28}$ The present study has demonstrated that diabetic conditions suppress leucocyte rolling and leucocyte accumulation in the retina during inflammation. Our findings are consistent with the preceding ones.

Our EIU models also supported the onset of anterior uveitis described in previous reports, with a peak at 24 hours after LPS injection. ${ }^{12}{ }^{1429}$ Anterior uveitis is the inflammation of anterior segment of the eye. A peak inflammation in the posterior segment of the eye was observed at 48 hours, which shifted backward in the time courses when compared with that in the anterior segment, as previously reported. ${ }^{14} 15$ Leucocyte rolling and adherence in the iris venules occur before infiltration of inflammatory cells in the AH. ${ }^{29}$ In our
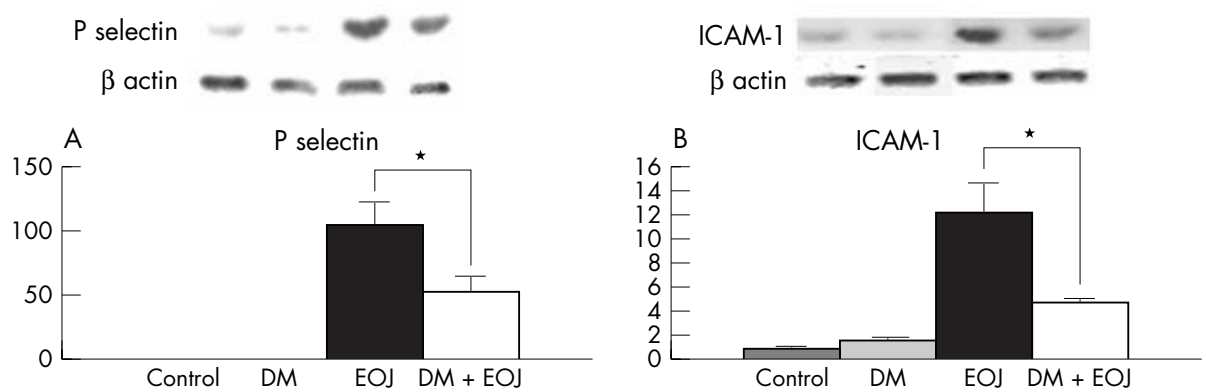

Figure 6 Gene expression of P-selectin (A), ICAM-I (B), TNF- $\alpha$ (C), and iNOS (D) 9 hours after LPS injection. Values are mean (SEM) $(n=6$ in both groups). ${ }^{*} \mathrm{p}<0.05$ compared with non-DM rats.
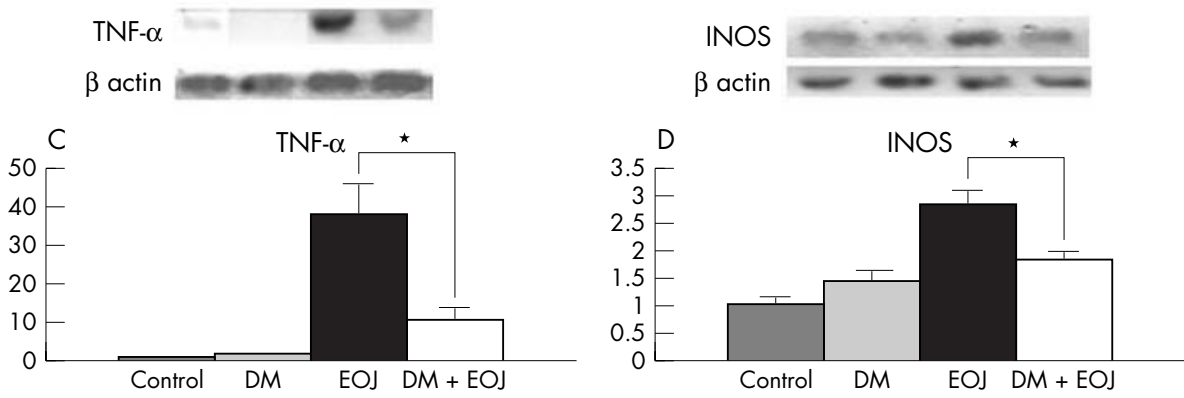


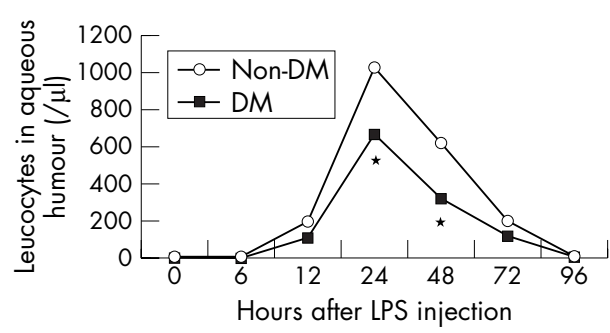

Figure 7 Time course of the number of leucocytes infiltrating into aqueous humour (AH) after LPS injection. Values are mean (SEM) $(n=6$ at each time point in both groups). ${ }^{*} p<0.05$ compared with non-DM rats.

diabetic groups the number of leucocytes in the AH was decreased, which implies reduced interactions between leucocytes and endothelial cells in the iris venules as in the retina.

Cell adhesion molecules, including ICAM-1 and P-selectin, are also involved in leucocyte-endothelial cell interactions. Their expression is reportedly reduced in the internal spermatic fascia of diabetic rats. ${ }^{8} \mathrm{P}$-selectin is a key mediator of leucocyte rolling along inflamed endothelial cells, ${ }^{30}$ and a previous study showed that P-selectin mediates leucocyte rolling EIU as well. ${ }^{31}$ Therefore, our finding of suppressed Pselectin gene expression might account for the reduced leucocyte rolling; diabetic conditions would suppress leucocyte rolling by inhibiting the expression of P-selectin on the endothelial cells of retinal veins. Increased expression of ICAM-1 is implicated in increased leucocyte adherence during EIU. ${ }^{32}$ The level of ICAM-1 mRNA expression in non-diabetic retinas after EIU induction was significantly upregulated compared with that in the diabetic retinas. This result would explain the reduced leucocyte accumulation of diabetic retina during EIU.

TNF- $\alpha$ is a major initial cytokine of systematic inflammation cascade, along with interleukin l (IL-1). TNF- $\alpha$ has been mentioned to upregulate the above mentioned adhesion molecules. It has been reported that the elevation of TNF- $\alpha$ expression stimulated by LPS in the bronchoalveolar lavage supernatant of diabetic rats was significantly reduced, ${ }^{9}$ and that TNF- $\alpha$ production by cultured peritoneal macrophages from diabetic rats after mineral oil injection was reduced. ${ }^{3}$ Our results also demonstrated that upregulation of TNF- $\alpha$ mRNA expression is significantly blocked in diabetic EIU rats. It is thus suggested that suppression of TNF- $\alpha$ mRNA expression leads to suppression of mRNA expression for adhesion molecules P-selectin and ICAM-1, and consequently to suppression of leucocyte rolling and leucocyte accumulation in the diabetic retina.

Vasodilation in the diabetic retina during EIU was reduced in our study. It was reported that hyperglycaemia decreases the bioavailability of NO and increases the synthesis of the vasoconstrictors, prostanoids and endothelin, via multiple mechanisms, which leads to impairment of endothelium dependent vascular relaxation. ${ }^{33}{ }^{34}$ In heart and liver, decreased activity and impaired induction of NO by LPS in STZ induced rat were also reported..$^{35}$ We demonstrated that diabetes reduced vein vasodilation in the EIU retina, probably caused by decreased expression of iNOS. This decreased expression of iNOS also explains the suppressed leucocyte rolling and accumulation, because of its effect on the expression of P-selectin and ICAM-1.22-24 The reason for suppressed iNOS expression in these cases was not clear, but suppressed TNF- $\alpha$ expression might be relevant to it, because LPS and TNF- $\alpha$ influence iNOS expression. ${ }^{36-38}$

In conclusion, we have demonstrated that leucocyteendothelial cell interactions in retinal tissue during EIU are impaired in diabetic rats, which may result from inhibited expression of adhesion molecules caused by suppressed TNF- $\alpha$ and iNOS expression. It is thus extrapolated that diabetic patients are vulnerable to infection and that, once an infection occurs it may be prolonged.

\section{ACKNOWLEDGEMENTS}

This work was supported by a grant from the Japan National Society for the Prevention of Blindness and a grant in aid for scientific research from the Japan Society for the Promotion of Science.

\section{Authors' affiliations}

H Tamura, J Kiryu, K Miyamoto, K Nishiijima, H Katsuta, S Miyahara, F Hirose, Y Honda, N Yoshimura, Department of Ophthalmology and Visual Sciences, Kyoto University Graduate School of Medicine, Kyoto, Japan

Competing interests: none declared

\section{REFERENCES}

1 Sugama Y, Tiruppathi C, offakidevi K, et al. Thrombin-induced expression of endothelial P-selectin and intercellular adhesion molecule-1: a mechanism for stabilizing neutrophil adhesion. J Cell Biol 1992;119:935-44.

2 Sluiter W, Pietersma A, Lamers JM, et al. Leukocyte adhesion molecules on the vascular endothelium: their role in the pathogenesis of cardiovascular disease and the mechanisms underlying their expression. J Cardiovasc Pharmacol 1993;4(22 Suppl):S37-44.

3 Doxey DL, Nares S, Park B, et al. Diabetes-induced impairment of macrophage cytokine release in a rat model: potential role of serum lipids. Life Sci 1998;63:1127-36

4 Fortes ZB, Farsky SP, Oliveira MA, et al. Direct vital microscopic study of defective leukocyte-endothelial interaction in diabetes mellitus. Diabetes 1991;40:1267-73.

5 Pereira MA, Sannomiya P, Leme JG. Inhibition of leukocyte chemotaxis by factor in alloxan-induced diabetic rat plasma. Diabetes 1987;36:1307-14

6 Sannomiya P, Pereira MA, Garcia-Leme J. Inhibition of leukocyte chemotaxis by serum factor in diabetes mellitus: selective depression of cell responses mediated by complement-derived chemoattractants. Agents Actions 1990;30:369-76.

7 Amano H, Yamamoto H, Senba M, et al. Impairment of endotoxin-induced macrophage inflammatory protein 2 gene expression in alveolar macrophages in streptozotocin-induced diabetes in mice. Infect Immun 2000;68:2925-9.

8 Cruz JW, Soto-Suazo MW, Hohman TC, et al. Minalrestat and leukocyte migration in diabetes mellitus. Diabetes Metab Res Rev 2003;19:223-31

9 Boichot E, Sannomiya P, Escofier N, et al. Endotoxin-induced acute lung injury in rats. Role of insulin. Pulm Pharmacol Ther 1999;12:285-90.

10 Yoshida M, Yoshimura N, Hangai M, et al. Interleukin-1 alpha, interleukin-1 beta, and tumor necrosis factor gene expression in endotoxin-induced uveitis. Invest Ophthalmol Vis Sci 1994;35:1107-13.

11 Rosenbaum JT, McDevitt HO, Guss RB, et al. Endotoxin-induced uveitis in rats as a model for human disease. Nature 1980;286:611-3.

12 Bhattacheriee P, Williams RN, Eakins KE. An evaluation of ocular inflammation following the injection of bacterial endotoxin into the rat foot pad. Invest Ophthalmol Vis Sci 1983;24:196-202.

13 Okumura A, Mochizuki M. Endotoxin-induced uveitis in rats: morphological and biochemical study. Jpn J Ophthalmol 1988;32:457-65.

14 Miyamoto K, Ogura Y, Hamada $M$, et al. In vivo quantification of leukocyte behavior in the retina during endotoxin-induced uveitis. Invest Ophthalmol Vis Sci 1996;37:2708-15.

15 Yamashiro K, Kiryu J, Tsujikawa A, et al. Inhibitory effects of antithrombin III against leukocyte rolling and infiltration during endotoxin-induced uveitis in rats. Invest Ophthalmol Vis Sci 2001;42:1553-60.

16 Tracey KJ, Beutler B, Lowry SF, et al. Shock and tissue injury induced by recombinant human cachectin. Science 1986;234:470-4.

17 Kettelhut IC, Fiers W, Goldberg AL. The toxic effects of tumor necrosis factor in vivo and their prevention by cyclooxygenase inhibitors. Proc Natl Acad Sci USA 1987;84:4273-7.

18 Vassalli P. The pathophysiology of tumor necrosis factors. Annu Rev Immunol 1992; 10:411-52.

19 Sanders WE, Wilson RW, Ballantyne CM, et al. Molecular cloning and analysis of in vivo expression of murine P-selectin. Blood 1992;80:795-800.

20 Koizumi K, Poulaki V, Doehmen S, et al. Contribution of TNF-alpha to leukocyte adhesion, vascular leakage, and apoptotic cell death in endotoxininduced uveitis in vivo. Invest Ophthalmol Vis Sci 2003;44:2184-91.

21 Mandai M, Yoshimura N, Yoshida M, et al. The role of nitric oxide synthase in endotoxin-induced uveitis: effects of NG-nitro L-arginine. Invest Ophthalmol Vis Sci 1994;35:3673-80.

22 Hierholzer C, Kalff JC, Billiar TR, et al. Induced nitric oxide promotes intestinal inflammation following hemorrhagic shock. Am J Physiol Gastrointest Liver Physiol 2004;286:G225-33.

23 Lush CW, Cepinskas G, Sibbald WJ, et al. Endothelial E- and P-selectin expression in iNOS-deficient mice exposed to polymicrobial sepsis. Am J Physiol Gastrointest Liver Physiol 2001;280:G291-7. 
24 Cuzzocrea S, Chatteriee PK, Mazzon E, et al. Role of induced nitric oxide in the initiation of the inflammatory response after postischemic injury. Shock 2002;18:169-76.

25 Nishiwaki H, Ogura Y, Kimura H, et al. Quantitative evaluation of leukocyte dynamics in retinal microcirculation. Invest Ophthalmol Vis Sci 1995; 36:123-30.

26 Miyahara S, Kiryu J, Tsujikawa A, et al. Argatroban attenuates leukocyte- and platelet-endothelial cell interactions after transient retinal ischemia. Stroke 2003;34:2043-9.

27 Lawrence MB, Springer TA. Leukocytes roll on a selectin at physiologic flow rates: distinction from and prerequisite for adhesion through integrins. Cell 1991;65:859-73.

28 Tsujikawa A, Kiryu J, Nonaka A, et al. Leukocyte-endothelial cell interactions in diabetic retina after transient retinal ischemia. Am J Physiol Regul Integr Comp Physiol 2000;279:R980-9.

29 Baatz H, Pleyer U, Thiel HJ, et al. In vivo study of leukocyte-endothelium interaction in endotoxin-induced uveitis. Invest Ophthalmol Vis Sci 1995:36:1960-7.

30 Mayadas TN, Johnson RC, Rayburn $\mathrm{H}$, et al. Leukocyte rolling and extravasation are severely compromised in P selectin-deficient mice. Cell 1993;74:541-54.

31 Miyamoto K, Ogura Y, Hamada M, et al. In vivo neutralization of P-selectin inhibits leukocyte-endothelial interactions in retinal microcirculation during ocular inflammation. Microvasc Res 1998:55:230-40.
32 Becker MD, Garman K, Whitcup SM, et al. Inhibition of leukocyte sticking and infiltration, but not rolling, by antibodies to ICAM-1 and LFA-1 in murine endotoxin-induced uveitis. Invest Ophthalmol Vis Sci 2001;42:2563-6

33 Tesfamariam B, Brown ML, Cohen RA. Elevated glucose impairs endotheliumdependent relaxation by activating protein kinase C. J Clin Invest 1991;87:1643-8

34 Creager MA, Luscher TF, Cosentino F, et al. Diabetes and vascular disease: pathophysiology, clinical consequences, and medical therapy: Part I. Circulation 2003:108:1527-32.

35 Khandelwal RL, Gupta D, Sulakhe PV. Decreased activity and impaired induction of nitric oxide synthase by lipopolysaccharides in streptozotocininduced diabetic rats. Biochim Biophys Acta 2003;1620:259-66.

36 Browner NC, Sellak H, Lincoln TM. Down-regulation of cyclic GMPdependent protein kinase expression by inflammatory cytokines in vascular smooth muscle cells. Am J Physiol Cell Physiol 2004;287:C88-96

37 Beck PL, Xavier R, Wong J, et al. Paradoxical roles of different nitric oxide synthase isoforms in colonic injury. Am J Physiol Gastrointest Liver Physiol 2004;286:G137-47.

38 Beasley D, Eldridge M. Interleukin-1 beta and tumor necrosis factor-alpha synergistically induce NO synthase in rat vascular smooth muscle cells. Am J Physiol 1994;266:R1197-203. 